Conclusions The inclusion of PGA $<0.5$ in the definition reduces the frequency of remission only in the long-term $(\geq 5$ year). A sustained remission, regardless of its definition, is associated with a lower chronic damage development. The addition of prednisone $\leq 5 \mathrm{mg} /$ day and/or PGA $<0.5$ to c-SLE$\mathrm{DAI}=0 / \leq 1$ increases the ability to predict the absence of damage accrual compared with $c S L E D A I=0 / \leq 1$ without substantial differences among them.
S1D:5 SLE DISEASE ACTIVITY INDEX GLUCOCORTICOSTEROID INDEX (SLEDAI-2KG) IDENTIFIES MORE RESPONDERS THAN SLEDAI-2K

${ }^{1} \mathrm{M}$ Urowitz, ${ }^{1} \mathrm{DD}$ Gladman, ${ }^{1} \mathrm{~J} \mathrm{Su},{ }^{1} \mathrm{NM}$ Anderson, ${ }^{2} \mathrm{Z}$ Touma. ${ }^{1}$ University of Toronto and Toronto Western Hospital, Department of Rheumatology, Toronto, Canada; ${ }^{2}$ University of Toronto, Department of Rheumatology, Institute of Health Policy, Management Evaluation, Toronto Western H, Toronto, Canada

10.1136/lupus-2018-abstract.5

Abstract S1D:4 Table 1 Proportion of patients achieving different levels of remission according to the duration of remission

\begin{tabular}{|c|c|c|c|c|c|c|}
\hline & $\begin{array}{l}\text { Unem } \\
\text { mited } \\
\text { pasiects }\end{array}$ & $\begin{array}{c}\text { I-yess } \\
\text { semissi } \\
\text { on }\end{array}$ & $\begin{array}{c}2-y e s s \\
\text { semissi } \\
\text { on }\end{array}$ & $\begin{array}{c}\text { 3-jess } \\
\text { semissi } \\
\infty\end{array}$ & $\begin{array}{c}4-j e s s \\
\text { semissi } \\
\text { on }\end{array}$ & $\begin{array}{c}x-y \text {-jess } \\
\text { semissi } \\
0 a\end{array}$ \\
\hline Remission Type & \multicolumn{6}{|c|}{ Nrenber (\%) of pasiens: } \\
\hline C-STEDAI $=0$ & $\begin{array}{c}29 \\
(9.9 \%)\end{array}$ & $\begin{array}{c}31 \\
(10.6 \%)\end{array}$ & $\begin{array}{c}40 \\
(13.7 \%)\end{array}$ & $\begin{array}{c}44 \\
(15.0 \%)\end{array}$ & $\begin{array}{c}23 \\
(7.5 \%)\end{array}$ & $\begin{array}{c}126 \\
(43.0 \%)\end{array}$ \\
\hline C-SLEDAIS 1 & $(8.2 \%)$ & $\begin{array}{c}29 \\
(9.9 \%)\end{array}$ & $\begin{array}{c}36 \\
(123 \%)\end{array}$ & $\begin{array}{c}40 \\
(13.7 \%)\end{array}$ & $\begin{array}{c}22 \\
(7.5 \%)\end{array}$ & $\begin{array}{c}142 \\
(48.5 \%)\end{array}$ \\
\hline $\begin{array}{c}\text { C-SLEDAI }=0 \text { asd predairose } \\
\text { \&s maby }\end{array}$ & $\begin{array}{c}35 \\
(11.9 \%)\end{array}$ & $\begin{array}{c}27 \\
(9.2 \%)\end{array}$ & $\begin{array}{c}45 \\
(15.7 \%)\end{array}$ & $\begin{array}{c}48 \\
(16.4 \%)\end{array}$ & $\begin{array}{c}24 \\
(3.2 \%)\end{array}$ & $\begin{array}{c}113 \\
(35.6 \%)\end{array}$ \\
\hline $\begin{array}{c}\text { C-SLEDAIsl and predaitose } \\
\text { sf ma day }\end{array}$ & $\begin{array}{c}35 \\
(11.9 \%)\end{array}$ & $\begin{array}{c}26 \\
(3.9 \%)\end{array}$ & $\begin{array}{c}44 \\
(15.0 \%)\end{array}$ & $\begin{array}{c}44 \\
(15.0 \%)\end{array}$ & $\begin{array}{c}23 \\
(7.5 \%)\end{array}$ & $\begin{array}{c}121 \\
(41.3 \%)\end{array}$ \\
\hline c-SLEDAI $=0$ and $P G A<0.5$ & $\begin{array}{c}75 \\
(25.6 \%)\end{array}$ & $\begin{array}{c}42 \\
(143 \%)\end{array}$ & (17.7\%) & $\begin{array}{c}45 \\
(16.4 \%)\end{array}$ & $(2.2 \%)$ & $(18.5 \%)$ \\
\hline C-SLEDAIsl and PGA $=0.5$ & $\begin{array}{c}73 \\
(24.9 \%)\end{array}$ & $\begin{array}{c}41 \\
(14.0 \%)\end{array}$ & $\begin{array}{c}51 \\
(17.4 \%)\end{array}$ & $\begin{array}{c}48 \\
(16.4 \%)\end{array}$ & $\frac{22}{(7.5 \%)}$ & $\begin{array}{c}59 \\
(19.5 \%)\end{array}$ \\
\hline 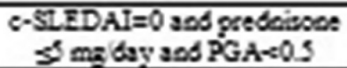 & $\begin{array}{c}\$ 2 \\
(28.0 \%)\end{array}$ & $\begin{array}{c}47 \\
(16.0 \%)\end{array}$ & $\begin{array}{c}55 \\
(18.5 \%)\end{array}$ & $\begin{array}{c}47 \\
(16.0 \%)\end{array}$ & $\begin{array}{c}13 \\
(6.1 \%)\end{array}$ & $\begin{array}{c}44 \\
(15.0 \%)\end{array}$ \\
\hline 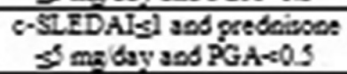 & $\begin{array}{c}90 \\
(27.3 \%)\end{array}$ & $\begin{array}{c}48 \\
(16.4 \%)\end{array}$ & $\begin{array}{c}53 \\
(18.1 \%)\end{array}$ & $\begin{array}{c}47 \\
(16.0 \%)\end{array}$ & $\begin{array}{c}19 \\
(65 \%)\end{array}$ & $\begin{array}{c}46 \\
(13.7 \%)\end{array}$ \\
\hline
\end{tabular}

Abstract S1D:4 Table 2 Multivariate analysis: predictors of damage accrual over the follow-up

\begin{tabular}{|c|c|c|c|c|c|c|c|c|}
\hline & C-SLEDAI $=0$ & C-STEDAI $\leq 1$ & $\begin{array}{c}\text { c-SLEDAI =0 } \\
\text { and } \\
\text { predoizoses s } \\
\text { mgday }\end{array}$ & $\begin{array}{c}\text { c-SLEDAI sl } \\
\text { and } \\
\text { predoiesos ss } \\
\text { mgday }\end{array}$ & $\begin{array}{l}\text { C-SLEDAI =0 } \\
\text { asd } P G A=0.5\end{array}$ & $\begin{array}{l}\text { C-SLEDAI } 51 \\
\operatorname{sed} P G A<0.5\end{array}$ & 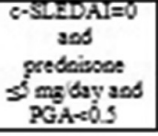 & $\begin{array}{c}\text { C-SLEDAISI } \\
\text { and } \\
\text { predaieses } \\
5 \text { maday and } \\
\text { PGA<0.s }\end{array}$ \\
\hline & \multicolumn{8}{|c|}{ Odds natio } \\
\hline 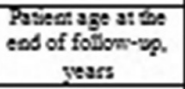 & $\begin{array}{c}1.035 \\
(1.012-1.054) \\
=0.004\end{array}$ & $\begin{array}{c}1.035 \\
(1.013-1.064) \\
g=0.003\end{array}$ & $\begin{array}{c}1.035 \\
(1.010-1.051) \\
g=0.005\end{array}$ & $\begin{array}{c}1.035 \\
(1.013-1.064) \\
g=0.003\end{array}$ & 28 & 28. & $2=$ & $2=$ \\
\hline $\begin{array}{c}\text { Diseave bycasion } \\
\text { years }\end{array}$ & $\begin{array}{c}1.045 \\
(1.005-1.099) \\
g=0.023\end{array}$ & $\begin{array}{c}1.048 \\
(1.005-1.090) \\
=0.023\end{array}$ & $\begin{array}{c}1.050 \\
(1.009-1.093) \\
g=0.018\end{array}$ & $\begin{array}{c}1.045 \\
(1.005-1.059) \\
g=0.028\end{array}$ & $\begin{array}{c}1.049 \\
(1.013-1.096) \\
=0.007\end{array}$ & $\begin{array}{c}1.048 \\
(1.013-1.095) \\
g=0.007\end{array}$ & $\begin{array}{c}2.041 \\
(1.002-1.052) \\
\rho=0.037\end{array}$ & $\begin{array}{c}1.041 \\
(1.001-1.052) \\
\rho=0.042\end{array}$ \\
\hline $\begin{array}{l}\text { Ansipbospbolipid } \\
\text { jyodrome }\end{array}$ & $\begin{array}{c}3.571 \\
(1.559-9.610) \\
j=0.004\end{array}$ & $\begin{array}{l}4.155 \\
(1.709- \\
10.103) \\
=0.002\end{array}$ & $\begin{array}{l}4.202 \\
(1.676- \\
10.536) \\
=0.002\end{array}$ & $\begin{array}{l}4.392 \\
(1.779- \\
10.947) \\
g=0.001\end{array}$ & $\begin{array}{c}3.257 \\
(1.330-8.122) \\
j=0.010\end{array}$ & $\begin{array}{c}3.122 \\
(1.297-7.510) \\
j=0.011\end{array}$ & $\begin{array}{c}3.042 \\
(1.469-3.493) \\
\delta=0.005\end{array}$ & $\begin{array}{c}3.436 \\
(1.423-8.293) \\
g=0.005\end{array}$ \\
\hline Vasculisis & $\begin{array}{l}3.950 \\
(1.357- \\
11.495) \\
g=0.012\end{array}$ & $\begin{array}{l}3.726 \\
(1.292- \\
10.742) \\
=0.015\end{array}$ & $\begin{array}{l}3.412 \\
(1.146- \\
10.156) \\
=0.027\end{array}$ & $\begin{array}{l}3.443 \\
(1166- \\
10.170) \\
=0.025\end{array}$ & $\begin{array}{c}3.354 \\
(1.156-9.732) \\
g=0.026\end{array}$ & $\begin{array}{c}3.162 \\
(1.1207- \\
\$ .929) \\
=0.030\end{array}$ & $\begin{array}{c}3.042 \\
(1.109-8.346) \\
=0.0315\end{array}$ & $\begin{array}{c}3.057 \\
(1.109-3.459) \\
g=0.031\end{array}$ \\
\hline$z^{2}$-geser remission & $\begin{array}{c}0.079 \\
(0.026-0.239) \\
-00.001\end{array}$ & $\begin{array}{c}0.115 \\
(0.035-0.377) \\
\sigma 0.001\end{array}$ & $\begin{array}{c}0.035 \\
(0.012-0.127) \\
\sigma 0.001\end{array}$ & $\begin{array}{c}0.044 \\
(0.013-0.144) \\
\approx 0.001\end{array}$ & $\begin{array}{c}0.055 \\
(0.021-0.142) \\
\sigma 0.001\end{array}$ & $\begin{array}{c}0.072 \\
(0.030-0.177) \\
\sigma 00.001\end{array}$ & $\begin{array}{c}0.073 \\
(0.0309- \\
0.203) \\
<0.001\end{array}$ & $\begin{array}{c}0.079 \\
(0.031-0.203) \\
\sigma=0.001\end{array}$ \\
\hline 4-year remission & $\begin{array}{c}0.190 \\
(0.049-0.727) \\
g-0.015\end{array}$ & $\begin{array}{c}0.205 \\
(0.049-0.594) \\
=0.033\end{array}$ & $\begin{array}{c}0.057 \\
(0.021-0.359) \\
=0.001\end{array}$ & $\begin{array}{c}0.053 \\
(0.020-0.354) \\
=0.001\end{array}$ & $\begin{array}{c}0.044 \\
(0.011-0.185) \\
\sigma 0.001\end{array}$ & $\begin{array}{c}0.070 \\
(0.019-0.255) \\
\approx 0.001\end{array}$ & $\begin{array}{c}0.100 \\
(0.029-0.339) \\
g-0.003\end{array}$ & $\begin{array}{c}0.079 \\
(0.021-0.295) \\
g-0.003\end{array}$ \\
\hline 3-yeas semission & $\begin{array}{l}0.21 \\
(0.055-0.751) \\
=0.016\end{array}$ & $\begin{array}{c}0.235 \\
(0.053-0.595) \\
=0.034\end{array}$ & $\begin{array}{c}0.115 \\
(0.033-0.413) \\
=0.001\end{array}$ & $\begin{array}{c}0.105 \\
(0.030-0.379) \\
=0.001\end{array}$ & $\begin{array}{c}0.3352 \\
(0.145-0.759) \\
=0.009\end{array}$ & $\begin{array}{c}0.355 \\
(0.047-0.775) \\
2-0.010\end{array}$ & $\begin{array}{c}0.214 \\
(0.094-0.490) \\
g-0.001\end{array}$ & $\begin{array}{c}0.163 \\
(0.069-0.393) \\
g=0.001\end{array}$ \\
\hline 2-yeser semission & $2 s$ & as. & $\begin{array}{c}0.265 \\
(0.075-0.959) \\
g=0.043\end{array}$ & $\begin{array}{c}0.234 \\
(0.079-1.023) \\
g=0.054\end{array}$ & $\begin{array}{c}0.274 \\
(0.122-0.615) \\
=0.002\end{array}$ & $\begin{array}{c}0.293 \\
(0.131-0.657) \\
=0.003\end{array}$ & $\begin{array}{c}0.275 \\
(0.126-0.603) \\
g=0.001\end{array}$ & $\begin{array}{c}0.257 \\
(0.130-0.636) \\
g=0.007\end{array}$ \\
\hline l-yeser semissios & as. & as. & $a s$ & $a=$ & as. & $a s$ & $a=$ & $\begin{array}{c}0.433 \\
(0.199-0.992) \\
g=0.048\end{array}$ \\
\hline No remission & 28. & as. & as. & as. & as. & as. & as. & as. \\
\hline
\end{tabular}


Background/purpose Systemic Lupus Erythematosus Disease Activity Index-2000 (SLEDAI-2K) is one of the most commonly used disease activity indices in clinical practice and research but this index doesn't account for severity within each descriptor. Moreover, in clinical trials, the use of standard of care (SoC), which includes glucocorticosteroid (GCS) often confounds trial results.

We developed and validated a novel lupus disease activity index, SLEDAI-2K GCS (SLEDAI-2KG), that describes disease activity while accounting for GCS dose. SLEDAI-2KG has the same descriptors as SLEDAI-2K in addition to a new descriptor 'GCS' with different weight scores based on the dose of GCS. Furthermore, SLEDAI-2KG has a low administration burden and a simple scoring system similar to SLEDAI-2K. We aimed to compare the performance of SLEDAI-2K and SGI in identifying responders in response to SoC.

Methods Patients have been followed prospectively according to a standard protocol between January 2011 and January 2014, at a single lupus centre, with active disease (SLEDAI$2 \mathrm{~K} \geq 6$ ), on prednisone $\geq 10 \mathrm{mg} / \mathrm{day}$, and with follow up visits within 5-24 months were studied. Treatment was determined based on the judgment of the treating rheumatologist.

Response to SoC therapy, at first follow up visit, was assessed by SLEDAI- $2 \mathrm{~K}$ and SLEDAI-2KG. Responders were defined based on the decrease in SLEDAI-2K and SGI score by $\geq 4$. The performance of SLEDAI-2K and SGI was also compared using different cut-off points; 5, 6 and 7. Descriptive analysis was used.

Results 111 patients met the inclusion criteria of the study and were further analysed. Patients' characteristics are represented in table 1 .

SLEDAI-2KG identified more responders at 6 months $(94 \%$ vs $84 \%)$ and at 12 months (92\% vs $76 \%$ ) compared to SLEDAI-2K by cut off of 4 . SLEDAI-2KG also identified more responders with cut off points 5, 6 and 7 (table 2).

Abstract S1D:5 Table 1 Patients characteristics

\begin{tabular}{|c|c|}
\hline Variables & Total $(n=111)$ \\
\hline Sex (lemale) & $98(88.3 \%)$ \\
\hline Age at baseline (mean $\div$ SD) & $35.75 \div 11.51$ \\
\hline SLF duration at baseline (mean \pm SD) & $9.02 \div 7.74$ \\
\hline \multicolumn{2}{|l|}{ Ethnikity } \\
\hline Asian & $13(11.76)$ \\
\hline Black & $23(20.779)$ \\
\hline Caucasian & $51(45.964)$ \\
\hline Others & $24(21.686)$ \\
\hline Months from baseline to 1 " follow up (mean \pm SD) & $7.63 \div 2.95$ \\
\hline SLEDM-2K at buseline (mean \pm SD) & $12.39 \div 6.03$ \\
\hline Prednisone dose at baseline $(\mathrm{mg} / \mathrm{day})$ (mean \pm SD) & $22.94 \pm 14.19$ \\
\hline SLEDAI-2KG at baseline $($ mean $\$ S D)$. & $17.48 \pm 6.78$ \\
\hline SLEDAI-2K at 1\% follow-up & $8.08 \div 6.04$ \\
\hline Prednisone dose at 1 " follow-up (mg/day) (mesn \pm SD) & $15.23 \pm 10.94$ \\
\hline SLEDAL-2KG at $1^{\text {"f follow-up }}$ & $12.67 \pm 6.98$ \\
\hline
\end{tabular}

Conclusion The novel index, SLEDAI-2KG, is superior to SLEDAI-2K in identifying responders at 6 and 12 months accounting for steroid dose and thus adjusting for severity within each descriptor of SLEDAI-2K. SLEDAI-2KG has the ability to enhance analyses in clinical trials to differentiate between responders on minimal and moderate/large doses of GCS.

Abstract S1D:5 Table 2 Responders by SLEDAI-2K and SLEDAI2KG in 111 patients

\begin{tabular}{|l|l|l|l|l|l|l|l|l|l|l|}
\hline Indices & \multicolumn{3}{|c|}{ Percentage of responders at 6 months } & \multicolumn{5}{|c|}{ Percentage of responders at 12 months } \\
\hline & 23 & 24 & 25 & 26 & 27 & 3 & 4 & 5 & 6 & 7 \\
\hline SLEDAL-2K & $34 \%$ & $84 \%$ & $65 \%$ & $68 \%$ & $59 \%$ & $77 \%$ & $76 \%$ & $57 \%$ & $55 \%$ & $44 \%$ \\
\hline SLEDAL-2KG & $95 \%$ & $94 \%$ & $88 \%$ & $83 \%$ & $79 \%$ & $93 \%$ & $92 \%$ & $80 \%$ & $30 \%$ & $6 \% \%$ \\
\hline $\begin{array}{l}\text { Additional } \\
\text { Responders }\end{array}$ & $11 \%$ & $10 \%$ & $20 \%$ & $15 \%$ & $20 \%$ & $16 \%$ & $16 \%$ & $23 \%$ & $15 \%$ & $18 \%$ \\
\hline
\end{tabular}

\section{S1D:6 TARGETING PLASMA CELLS AND THEIR PRECURSORS BY IMMUNOABLATION VERSUS BORTEZOMIB PLUS RITUXIMAB IN SYSTEMIC LUPUS ERYTHEMTOSUS}

${ }^{1} \mathrm{~T}$ Alexander, ${ }^{1} \mathrm{~B}$ Hoyer, ${ }^{1} \mathrm{Q}$ Cheng, ${ }^{1} \mathrm{~L}$ Khodadadi, ${ }^{1} \mathrm{~A}$ Taddeo, ${ }^{2} \mathrm{~J}$ Klotsche, ${ }^{3} \mathrm{~A}$ Thiel,

${ }^{1} \mathrm{G}$ Burmester, ${ }^{2} \mathrm{~A}$ Radbruch, ${ }^{4} \mathrm{R}$ Arnold, ${ }^{1} \mathrm{~F}$ Hiepe. ${ }^{1}$ Charité University Medicine, Department of Rheumatology, Berlin, Germany; 'Deutsches Rheuma-Forschungszentrum, Berlin, Germany; ${ }^{3}$ Charité University Medicine, Berlin-Brandenburg Centre for Regenerative Therapies, Berlin, Germany; ${ }^{4}$ Charité University Medicine Berlin, Medical Department, Division of Haematology, Oncology and Tumour Immunology

\subsection{6/lupus-2018-abstract.6}

Purpose To investigate the therapeutic relevance of targeting long-lived plasma cells (PC), which contribute to the chronicity of SLE through continuous secretion of pathogenic antibodies, using immunoablation with antithymocyte-globulin (ATG) in the context of haematopoietic stem cell transplantation (HSCT) or proteasome inhibition with Bortezomib.

Methods Prospective analysis of outcome in 10 SLE patients after receiving autologous HSCT between 1998 and 2012 and 8 SLE patients after receiving a median 2 cycles (range 1-4) of Bortezomib $1.3 \mathrm{mg} / \mathrm{m} 2$ between 2009 and 2012 at the Charité - University Medicine Berlin. Multiparametric flow cytometry was applied to characterise peripheral blood or bone marrow PC subsets and B cells. Autoantibodies and vaccine titres were investigated with ELISA.

Results In all HSCT treated patients clinical remissions (SLEDAI <3) were achieved, accompanied by a complete normalisation of anti-dsDNA antibody titres $(92.6 \%$ reduction) and a significant reduction of antinuclear antibodies and vaccine titres (measles 82.3\%). Peripheral blood B and PCs were virtually absent and bone marrow PCs largely depleted $(97.6 \%$ reduction, $n=1$ ) shortly after HSCT and regenerating $B$ cells almost exclusively displayed a naïve phenotype. Upon proteasome inhibition, clinical improvements were associated with a significant reduction of anti-dsDNA autoantibodies (69.3\%) and vaccine titers (measles $32.5 \%$ ). While B cell number/phenotype remained stable, both bone marrow and circulating PC were significantly reduced $(\sim 50 \%)$ but rapidly regenerated after proteasome inhibition, which could be prevented by additional rituximab therapy in one patient applied. 